ESAIM: COCV 19 (2013) 931-946

DOI: $10.1051 / \mathrm{cocv} / 2012039$
ESAIM: Control, Optimisation and Calculus of Variations

www.esaim-cocv.org

\title{
HOMOGENIZATION AT DIFFERENT LINEAR SCALES, BOUNDED MARTINGALES AND THE TWO-SCALE SHUFFLE LIMIT
}

\author{
KÉvin SAntugini ${ }^{1,2,3}$
}

\begin{abstract}
In this paper, we consider two-scale limits obtained with increasing homogenization periods, each period being an entire multiple of the previous one. We establish that, up to a measure preserving rearrangement, these two-scale limits form a martingale which is bounded: the rearranged two-scale limits themselves converge both strongly in $\mathrm{L}^{2}$ and almost everywhere when the period tends to $+\infty$. This limit, called the Two-Scale Shuffle limit, contains all the information present in all the two-scale limits in the sequence.
\end{abstract}

Mathematics Subject Classification. 35B27.

Received September 15, 2011. Revised June 7, 2012

Published online July 4, 2013.

\section{INTRODUCTION}

Homogenization is used to study the solutions to equations when there are multiple scales of interest, usually a microscopic one and a macroscopic one. In particular, one may consider the solutions $u_{\varepsilon}$ to a partial differential equation with locally $\varepsilon$-periodic coefficients and study their behavior as the small period $\varepsilon$ tends to 0 . Twoscale convergence, introduced by Nguetseng [10] and Allaire [1], is suited to study this particular subset of homogenization problems called periodic homogenization. It was later extended to the case of periodic surfaces by Neuss Radu $[8,9]$ and Allaire, Damlamian and Hornung [2]. It can also be used in the presence of periodic holes in the geometry, see $[5,6]$ or to homogenize multilayers $[12,13]$.

Intuitively, two-scale convergence introduces the concept of two-scale limit $u_{0}$ which is a function of both a macroscopic variable $\boldsymbol{x}$ - also called slow variable - and a microscopic p-periodic variable $\boldsymbol{y}$ - also called fast variable - such that, in some "meaning", $\boldsymbol{x} \mapsto u_{0}(\boldsymbol{x}, \boldsymbol{x} / \varepsilon)$ is a good approximation of $u_{\varepsilon}$.

As indicated by its name, two-scale convergence captures the behavior at two scales: the macroscopic one and the $p \varepsilon$-periodic one. However, two-scale convergence does not capture all phenomena that happens at a scale linear in $\varepsilon$ but only those whose length scale is $p \varepsilon / m$ where $m$ is an integer. The two-scale limit of a sequence depends not only on the asymptotic scale, but also on the precise value of the chosen period. For example, any phenomena happening at the length scale of $2 \varepsilon$ will not be fully apparent in the two-scale limit computed with period $\varepsilon$. The two-scale limit computed with period $2 \varepsilon$ will contain no less - and might actually

Keywords and phrases. Homogenization, two-scale convergence.

1 University Bordeaux, IMB, UMR 5251, 33400 Talence, France. Kevin.Santugini@math.u-bordeaux1.fr

2 CNRS, IMB, UMR 5251, 33400 Talence, France.

3 INRIA, 33400 Talence, France. 
contain more - information than the two-scale limit computed with period $\varepsilon$. For example, the homogenization of $\sin (2 \pi x / \varepsilon)+\sin (\pi x / \varepsilon)$ gives a two-scale limit of $u_{0}:(x, y) \mapsto \sin (2 \pi y)$ if computed with the homogenization period $\varepsilon$, i.e., when $p=1$, and $u_{0}:(x, y) \mapsto \sin (2 \pi y)+\sin (\pi y)$ if computed with the homogenization period $2 \varepsilon$, i.e., when $p=2$. Furthermore, if we choose $p=1 / 2$, then the two-scale limit is none other than the null function. Worse, the scale factor $p$ could be irrational.

The choice of the scale factor $p$ used in the homogenization process is therefore of utmost importance in two-scale convergence. Using a badly chosen scale factor $p$ may and will often cause a huge loss of information. At worst, we recover no more information than the one obtained by the standard weak $\mathrm{L}^{2}$ limit: if $p \varepsilon$ is the correct choice of homogenization period, the two-scale limit computed with period $\lambda p \varepsilon$ where $\lambda$ is an irrational number should, intuitively, carry no information about what happens at scale $p \varepsilon$.

Fortunately, there is usually a natural choice of period: the coefficients of the partial differential equation are often chosen locally $\varepsilon$-periodic. The most natural choice is to choose $p=1$, i.e., to consider the correct microscopic scale for $u_{\varepsilon}$ is $\varepsilon$ itself. If there are two important periods to consider $p \varepsilon$ and $p^{\prime} \varepsilon$, the intuitive solution is to choose a period that is an entire multiple of both. However, this can only be done if the ratio $p / p^{\prime}$ between the two scale factors is a rational number.

When the two-scale limit depends on the fast variable, we may consider an homogenization period of $p_{2} \varepsilon$ instead of $p_{1} \varepsilon$ where $p_{2} / p_{1}$ is a positive integer. The two-scale limit computed with the homogenization period $p_{2} \varepsilon$ contains more information than the two-scale limit computed with the homogenization period $p_{1} \varepsilon$. It is then natural to study the behavior of the two-scale limit as the scale factor tends to $+\infty$. Allaire and Conca studied in [3] a similar problem and established, for an elliptic problem, the behavior of the spectra of the equation satisfied by the two-scale limit as the scale factor $p$ goes to $+\infty$. Ben Arous and Owhadi [4] studied the behavior of the Brownian motion in a periodic potential using multiscale homogenization when the ratio between two successive scales is bounded from above and below.

In this paper, we consider various two-scale limits, each computed with a different homogenization period. In particular, we consider a sequence of periods $\left(p_{n}\right)_{n \in \mathbb{N}}$ such that for all integers $n, p_{n+1} / p_{n}$ is a positive integer and we study the two-scale limit of $\left(u_{\varepsilon}\right)_{\varepsilon>0}$ computed with the homogenization period $p_{n} \varepsilon$. This two-scale limit, denoted $u_{0, p_{n}}$, is $p_{n}$-periodic in each component of its fast variable. Since $p_{n+1}$ is always an entire multiple of $p_{n}$, one can always recover the two-scale limit $u_{0, p_{n}}$ from the two scale limit $u_{0, p_{n+1}}$. If $p_{n+1}=m_{n} p_{n}$ and in dimension $d \geq 1$ :

$$
u_{0, p_{n}}(\boldsymbol{x}, \boldsymbol{y})=\frac{1}{m_{n}^{d}} \sum_{\boldsymbol{\alpha} \in \llbracket 0, m_{n}-1 \rrbracket^{d}} u_{0, p_{n+1}}\left(\boldsymbol{x}, \boldsymbol{y}+p_{n} \boldsymbol{\alpha}\right)
$$

The sequence of two-scale limits $\left(u_{0, p_{n}}\right)_{n \in \mathbb{N}}$ yields increasing information on the asymptotic behavior of $\left(u_{\varepsilon}\right)_{\varepsilon>0}$. A natural question is whether the two-scale limits $u_{0, p_{n}}$ themselves converge whenever $n$ tends to $+\infty$. I.e., does there exist a function that carry the information of all the $p_{n}$-two-scale limits? The goal of our paper is to answer this question. The answer is positive. We show in this paper that the sequence of two-scale limits is, after a measure preserving rearrangement, a bounded martingale in $\mathrm{L}^{2}$ and therefore converges both strongly in $\mathrm{L}^{2}$ and almost everywhere to a function we call the Two-Scale Shuffle limit.

In Section 2, we remind the reader of previously known results: two-scale convergence and the convergence properties of bounded martingales. In Section 3, we show how the different two-scale limits are related to each other through martingale-like equalities and explain how to transform these two-scale limits to get a genuine martingale. This leads to our stating of our main theorem: Theorem 3.8 in which we show that in a certain meaning the two-scale limits themselves converge to the Two-Scale Shuffle limit. In addition, we also state in Corollary 3.9 that all the information present in all the two-scale limits is contained in the Two-Scale Shuffle limit. In Section 4, we use this result on the heat equation in multilayers with transmission conditions between adjacent layers and establish, for this particular example, the equation satisfied by the Two-Scale Shuffle limit in Theorem 4.1. 


\section{Notations, PRerequisites And KNOWn RESUlts}

Throughout this paper, if $x$ is in $\mathbb{R}$, we denote by $\lfloor x\rfloor$ the integer part of $x$. We also denote by $\llbracket n_{1}, n_{2} \rrbracket$ the set $\left[n_{1}, n_{2}\right] \cap \mathbb{N}$. To make the present paper as self-contained as possible, we recall in this section known results on the two main mathematical tools we use to prove our main theorem: two-scale convergence in Section 2.1, and classical results on the convergence of bounded martingales in Section 2.2.

\subsection{The classical notion of two-scale convergence}

First, as in [1], we introduce some notations. In this paper, $p$ always refer to a scale factor. It remains constant while taking the two-scale limit. However, the goal of this paper is to observe the behavior of the two-scale limits as $p$ tends to $+\infty$.

By $\Omega$, we denote a bounded open domain of $\mathbb{R}^{d}$ where $d \geq 1$. By $Y_{p}$, we denote the cube $[0, p]^{d}$. By $\mathrm{L}_{\#}^{2}\left(Y_{p}\right)$, we denote the space of measurable functions defined over $\mathbb{R}^{d}$, that are $p$-periodic in each variable and that are square integrable over $Y_{p}$. By $\mathcal{C}_{\#}\left(Y_{p}\right)$, we denote the set of continuous functions defined on $\mathbb{R}^{d}$ that are $p$-periodic in each variable.

We reproduce the now classical definition of two-scale convergence found in $[1,10]$. For convenience, we added the scale factor $p$.

Definition 2.1 (two-scale convergence). Let $p$ be a positive real. A sequence $\left(u_{\varepsilon}\right)_{\varepsilon>0}$ belonging to $\mathrm{L}^{2}(\Omega)$ is said to $p$-two-scale converge if there exists $u_{0, p}$ in $\mathrm{L}^{2}\left(\Omega \times Y_{p}\right)$ such that:

$$
\lim _{\varepsilon \rightarrow 0} \int_{\Omega} u_{\varepsilon}(\boldsymbol{x}) \psi\left(\boldsymbol{x}, \frac{\boldsymbol{x}}{\varepsilon}\right) \mathrm{d} \boldsymbol{x}=\frac{1}{p^{d}} \int_{\Omega} \int_{Y_{p}} u_{0, p}(\boldsymbol{x}, \boldsymbol{y}) \psi(\boldsymbol{x}, \boldsymbol{y}) \mathrm{d} \boldsymbol{y} \mathrm{d} \boldsymbol{x},
$$

for all $\psi$ in $\mathrm{L}^{2}\left(\Omega ; \mathcal{C}_{\#}\left(Y_{p}\right)\right)$.

It is a common abuse of notation to also designate by $u_{0, p}$ the unique extension of $u_{0, p}$ to $\Omega \times \mathbb{R}^{d}$ that is $p$-periodic in the last $d$ variables.

Allaire, see [1], and Nguetseng, see [10], proved that any sequence of functions bounded in $\mathrm{L}^{2}$ has a subsequence that two-scale converges. Let's reproduce this precise compactness result.

Theorem 2.2. Let $\left(u_{\varepsilon}\right)_{\varepsilon>0}$ be a sequence of functions bounded in $\mathrm{L}^{2}(\Omega)$. Then, there exist $u_{0, p}$ in $\mathrm{L}^{2}(\Omega \times] 0, p\left[{ }^{d}\right)$ and a subsequence $\varepsilon_{k}$ converging to 0 such that

$$
\lim _{k \rightarrow \infty} \int_{\Omega} u_{\varepsilon_{k}}(\boldsymbol{x}) \psi\left(\boldsymbol{x}, \frac{\boldsymbol{x}}{\varepsilon_{k}}\right) \mathrm{d} \boldsymbol{x}=\frac{1}{p^{d}} \int_{\Omega} \int_{Y_{p}} u_{0, p}(\boldsymbol{x}, \boldsymbol{y}) \psi(\boldsymbol{x}, \boldsymbol{y}) \mathrm{d} \boldsymbol{y} \mathrm{d} \boldsymbol{x},
$$

for all $\psi$ in $\mathrm{L}^{2}\left(\Omega ; \mathcal{C}_{\#}\left(Y_{p}\right)\right)$.

Proof. See Allaire [1], Theorem 1.2 and Nguetseng [10], Theorem 2. The presence of the scale factor $p$ has no impact on the proof.

We also have the classical proposition

Proposition 2.3. Let $u_{\varepsilon} p$-two-scale converges to $u_{0, p}$. Then,

$$
\frac{1}{p^{d / 2}}\left\|u_{0, p}\right\|_{\mathrm{L}^{2}\left(\Omega \times Y_{p}\right)} \leq \liminf _{\varepsilon \rightarrow 0}\left\|u_{\varepsilon}\right\|_{\mathrm{L}^{2}(\Omega)} .
$$

Proof. See Allaire [1], Proposition 1.6. The presence of the scale factor $p$ has no impact on the proof.

The next proposition is easy to derive from Theorem 2.2. 
Proposition 2.4. Let $\left(p_{n}\right)_{n \in \mathbb{N}}$ be an increasing sequence of positive real numbers. Let $\left(u_{\varepsilon}\right)_{\varepsilon>0}$ be a sequence of functions bounded in $\mathrm{L}^{2}(\Omega)$. Then, there exist a subsequence $\left(\varepsilon_{k}\right)_{k \in \mathbb{N}}$ converging to 0 , and a sequence of functions $u_{0, p_{n}}$ in $\left.\mathrm{L}^{2}(\Omega \times] 0, p_{n}{ }^{d}\right)$ such that, for any non-negative integer $n$, the sequence $\left(u_{\varepsilon_{k}}\right)_{k \in \mathbb{N}} p_{n}$-two-scale converges to $u_{0, p_{n}}$. I.e., such that for all integers $n$ :

$$
\lim _{k \rightarrow \infty} \int_{\Omega} u_{\varepsilon_{k}}(\boldsymbol{x}) \psi\left(\boldsymbol{x}, \frac{\boldsymbol{x}}{\varepsilon_{k}}\right) \mathrm{d} \boldsymbol{x}=\frac{1}{p_{n}^{d}} \int_{\Omega} \int_{Y_{p_{n}}} u_{0, p_{n}}(\boldsymbol{x}, \boldsymbol{y}) \psi(\boldsymbol{x}, \boldsymbol{y}) \mathrm{d} \boldsymbol{y} \mathrm{d} \boldsymbol{x},
$$

for all $\psi$ in $\mathrm{L}^{2}\left(\Omega ; \mathcal{C}_{\#}\left(Y_{p_{n}}\right)\right)$.

Proof. Apply Theorem 2.2 multiple times and proceed via diagonal extraction.

Our goal in this paper is to study the limit of $u_{0, p_{n}}$ as $p_{n}$ tends to $+\infty$.

\subsection{Convergence of bounded martingales}

In this section, we recall the notions of probability theory needed to prove our main theorem. In particular, we are interested in using the convergence properties of bounded martingales. For more details, the reader may consult [7]. We assume the reader to be familiar with the notions of $\sigma$-field and $\sigma$-additivity in measure theory.

We use the following common notations:

- If $C$ is a subset of $\mathcal{P}(X)$, we denote by $\sigma(C)$ the smallest $\sigma$-field in $X$ that contains $C$.

- If $D$ is a topological space, we denote by $\mathcal{B}(D)$ the set of all Borel sets in $D$, i.e., the smallest $\sigma$-field containing all the open subsets of $D$.

Definition 2.5 (measurable space). A pair $(X, \mathcal{F})$ is said to be a measurable space if $\mathcal{F}$ is a $\sigma$-field in $X$.

Definition 2.6 (measure space). A triplet $(X, \mathcal{F}, \mu)$ is said to be a measure space if $(X, \mathcal{F})$ is a measurable space and if $\mu$ is a positive $\sigma$-additive measure on $(X, \mathcal{F})$.

A measure space $(X, \mathcal{F}, \mu)$ is said to be finite if $\mu(X)<+\infty$. A measure space $(X, \mathcal{F}, \mu)$ is said to be $\sigma$-finite if $X$ is the countable union of $\mathcal{F}$-measurable sets of finite measure. A measure space $(X, \mathcal{F}, \mathbb{P})$ is said to be a probability space if $\mathbb{P}(X)=1$.

We start by recalling the definition of conditional expectation, see [7] (Chapter 6, Thm. 6.1) for more details. Usually, the conditional expectation is defined for probability spaces. The definition extends without problem to finite measure spaces and even, to some extent, to $\sigma$-finite measure spaces.

Definition 2.7 (conditional expectation). Let $(X, \mathcal{F}, \mu)$ be a measure space with $\mu$ being positive and $\sigma$-additive. Let $\mathcal{G}$ be a $\sigma$-field such that $\mathcal{G} \subset \mathcal{F}$ and $(X, \mathcal{G}, \mu)$ is also $\sigma$-finite. Let $f: X \rightarrow \mathbb{R}$ be $\mathcal{F}$-measurable and in $L_{\text {loc }}^{1}(X, \mu)$. The conditional expectation of $f$ with respect to the $\sigma$-field $\mathcal{G}$ is denoted by $\mathbb{E}(f \mid \mathcal{G})$, and is defined as the unique, up to a modification on a set of null measure, $\mathcal{G}$-measurable function $g$ such that

$$
\int_{B} g(\omega) \mathrm{d} \omega=\int_{B} f(\omega) \mathrm{d} \omega,
$$

for all $B$ in $\mathcal{G}$.

The existence of the conditional expectation is given by Radon-Nikodym theorem. The measure $\mu$ need not be a probability measure. However, to apply Radon-Nykodim theorem, $(X, \mathcal{G}, \mu)$ needs to be $\sigma$-finite, hence the restriction in the definition. A statement and a proof of the Radon-Nikodym theorem can be found in [11], Theorem 6.10.

It is not enough that $(X, \mathcal{F}, \mu)$ be $\sigma$-finite in Definition 2.7.

Remark 2.8. When $\mathcal{G} \subset \mathcal{F}$, it does not follow from $(X, \mathcal{F}, \mu)$ being $\sigma$-finite that $(X, \mathcal{G}, \mu)$ is also $\sigma$-finite. A counter-example is easily obtained by setting $\mathcal{G}:=\{\emptyset, X\}$ whenever $\mu(X)=+\infty$. 
In our main theorem, we restrict ourselves to the case of finite measures. However, Remark 2.8 will explain why the martingale approach doesn't quite work for the most natural attempt to define a convergence for two-scale limits, see Section 3.1.

In order to define martingales, we remind the reader of the definition of filtration. We limit ourselves to filtrations indexed by the set $\mathbb{N}$. See [7], chapter 7, p. 120, for more details.

Definition 2.9 (filtrations). Let $(X, \mathcal{F})$ be a measurable space. A sequence $\left(\mathcal{F}_{n}\right)_{n \in \mathbb{N}}$ of $\sigma$-fields, $\mathcal{F}_{n} \subset \mathcal{F}$ is a filtration if, for all non-negative integers $n, \mathcal{F}_{n}$ is a subset of $\mathcal{F}_{n+1}$.

We now recall the definition of martingales.

Definition 2.10 (martingales). Let $(X, \mathcal{F}, \mu)$ be a $\sigma$-finite measure space. Let $\left(\mathcal{F}_{n}\right)_{n \in \mathbb{N}}$ be a filtration on $(X, \mathcal{F}, \mu)$ such that $\left(X, \mathcal{F}_{0}, \mu\right)$ is $\sigma$-finite.

A sequence $\left(f_{n}\right)_{n \in \mathbb{N}}$ is said to be a $\left(\mathcal{F}_{n}\right)_{n \in \mathbb{N}}$-martingale, if for all non-negative integers $n$ and $j$,

$$
f_{n}=\mathbb{E}\left(f_{n+j} \mid \mathcal{F}_{n}\right) .
$$

I.e., if $f_{n}$ is $\mathcal{F}_{n}$-measurable and if for all $F$ in $\mathcal{F}_{n}$ :

$$
\int_{F} f_{n}(\omega) \mathrm{d} \omega=\int_{F} f_{n+j}(\omega) \mathrm{d} \omega .
$$

We now reproduce the convergence results of bounded martingales:

Theorem 2.11 (convergence of bounded martingales). Let $(X, \mathcal{F}, \mu)$ be a measure space with finite measure. Let $\left(\mathcal{F}_{n}\right)_{n \in \mathbb{N}}$ be a filtration on the measurable space $(X, \mathcal{F})$. Let q be in $] 1,+\infty\left[\right.$. Let $\left(f_{n}\right)_{n \in \mathbb{N}}$ be a $\left(\mathcal{F}_{n}\right)_{n \in \mathbb{N}^{-}}$ martingale such that the sequence $\left(f_{n}\right)_{n \in \mathbb{N}}$ is bounded in $L^{q}(X)$. Then, the sequence $\left(f_{n}\right)_{n \in \mathbb{N}}$ converges both almost everywhere and strongly in $L^{q}(X, \mathbb{P})$.

Proof. See [7], Corollary 7.22 for the strong $L^{q}$ convergence. The almost everywhere convergence is stated in [7], Theorem 7.18 and holds even for $q=1$. While these two results are stated for probability measures, the finite measures case is easily deduced from the probability measure case by considering the probability measure $\mu(\cdot) / \mu(X)$.

The above theorem extends, at least partially, to $\sigma$-finite measures:

Remark 2.12. In Theorem 2.11, if the probability space $(X, \mathcal{F}, \mathbb{P})$ is replaced with $\sigma$-finite measure space $(X, \mathcal{F}, \mu)$ such that $\left(X, \mathcal{F}_{0}, \mu\right)$ is also $\sigma$-finite, then the bounded martingales converge almost everywhere and at least in $L_{l o c}^{q}$. It is unknown to the author if the strong $L^{q}$ convergence can be generalized to the $\sigma$-finite case.

\section{TWO-SCALE LIMITS AND BOUNDED MARTINGALES}

In this section, we always assume both of the following assumptions are satisfied:

Assumption 3.1 (integer scale ratios). We are given a real sequence $\left(p_{n}\right)_{n \in \mathbb{N}}$, such that for all $n$ in $\mathbb{N}, p_{n}>0$ and $p_{n+1}$ is an entire multiple of $p_{n}$. Moreover, we set for $n \geq 1, m_{n}:=p_{n} / p_{n-1} \in \mathbb{N}$, and for $n \geq 0$, $M_{n}:=p_{n} / p_{0} \in \mathbb{N}$.

Assumption 3.2. We are given a sequence of functions $\left(u_{\varepsilon}\right)_{\varepsilon>0}$ bounded in $\mathrm{L}^{2}(\Omega)$ and a decreasing sequence of positive $\left(\varepsilon_{k}\right)_{k \in \mathbb{N}}$ such that the sequence $\left(u_{\varepsilon_{k}}\right)_{k \in \mathbb{N}} p_{n}$-two-scale converges for all integers $n$ to a function $u_{0, p_{n}}$ that belongs to $\left.\mathrm{L}^{2}(\Omega \times] 0, p_{n}{ }^{{ }^{d}}\right)$.

This last assumption is justified by Proposition 2.4.

Our goal is to study the convergence of the two-scale limits $u_{0, p_{n}}$ when $n$ goes to infinity. In this section, we proceed as follows: we begin by establishing a useful equality that looks like a martingale equality in Section 3.1, then we propose a rearrangement of the two-scale limits in Section 3.2, and finally propose another rearrangement of the two-scale limits in Section 3.3, the shuffle, which transform the sequence of two-scale limits into a bounded martingale. 


\subsection{An almost martingale equality}

We start with a simple but essential proposition.

Proposition 3.3. Suppose both assumptions 3.1 and 3.2 are satisfied. Then, for all $j$ in $\mathbb{N}$, all $n$ in $\mathbb{N}$, almost all $\boldsymbol{x}$ in $\Omega$ and almost all $\boldsymbol{y}$ in $Y_{p}$ :

$$
u_{0, p_{n}}(\boldsymbol{x}, \boldsymbol{y})=\left(\frac{p_{n}}{p_{n+j}}\right)^{d} \sum_{\boldsymbol{\alpha} \in \llbracket 0, p_{n+j} / p_{n}-1 \rrbracket^{d}} u_{0, p_{n+j}}\left(\boldsymbol{x}, \boldsymbol{y}+\boldsymbol{\alpha} p_{n}\right) .
$$

Proof. Let $\phi$ belong to $\mathcal{C}^{\infty}\left(\bar{\Omega} \times \mathbb{R}^{d}\right)$ be $p_{n}$-periodic in the last $d$ variables. Since $p_{n+j} / p_{n}$ is an integer, $\phi$ is also $p_{n+j}$-periodic in the last $d$ variables. We take the limit of $\int_{\Omega} u_{\varepsilon}(\boldsymbol{x}) \phi(\boldsymbol{x}, \boldsymbol{x} / \varepsilon) \mathrm{d} \boldsymbol{x}$, as $\varepsilon$ tends to 0 , in the sense of two-scale convergence for both scale factors $p_{n+j}$ and $p_{n}$ :

$$
\begin{aligned}
\frac{1}{p_{n}^{d}} \int_{\Omega} \int_{Y_{p_{n}}} u_{0, p_{n}}(\boldsymbol{x}, \boldsymbol{y}) \phi(\boldsymbol{x}, \boldsymbol{y}) \mathrm{d} \boldsymbol{y} \mathrm{d} \boldsymbol{x} & =\frac{1}{p_{n+j}^{d}} \int_{\Omega} \int_{Y_{p_{n+j}}} u_{0, p_{n+j}}(\boldsymbol{x}, \boldsymbol{y}) \phi(\boldsymbol{x}, \boldsymbol{y}) \mathrm{d} \boldsymbol{y} \mathrm{d} \boldsymbol{x} \\
& =\frac{1}{p_{n+j}^{d}} \int_{\Omega} \int_{Y_{p_{n}}}\left(\sum_{\boldsymbol{\alpha} \in\left(\llbracket 0, p_{n+j} / p_{n}-1 \rrbracket^{d}\right.} u_{0, p_{n+j}}(\boldsymbol{x}, \boldsymbol{y}+\boldsymbol{\alpha} p)\right) \phi(\boldsymbol{x}, \boldsymbol{y}) \mathrm{d} \boldsymbol{y} \mathrm{d} \boldsymbol{x} .
\end{aligned}
$$

The most natural approach is to consider the $u_{0, p_{n}}$ as functions defined over $\Omega \times \mathbb{R}^{d}$ and to study their convergence in some meaning in $\Omega \times \mathbb{R}^{d}$. Such a convergence result would be ideal as the intuitive meaning of the limit would be easy to grasp. Equality (3.1) is similar to the martingale defining equality (2.3). Would it be possible to use the classical convergence properties of martingales, see Theorem 2.11, to prove the existence of a limit to the $u_{0, p_{n}}$ ? Unfortunately, the martingale approach doesn't work in this setting but the attempt is, nevertheless, instructive. First, we try to construct a filtration $\left(\mathcal{F}_{n}\right)_{n \in \mathbb{N}}$ for the $u_{0, p_{n}}$. For all positive integer $n$, the $u_{0, p_{n}}$ are $p_{n}$-periodic with respect to the last $d$ variables. Let $\mathcal{F}_{n}$ be the set of all Borel subsets of $\Omega \times \mathbb{R}^{d}$ that are invariant by translation of $\pm p_{n}$ along any of the last $d$ directions of $\Omega \times \mathbb{R}^{d}$. Clearly, $u_{0, p_{n}}$ is $\mathcal{F}_{n}$-measurable. However, the measure space $\left(\Omega \times \mathbb{R}^{d}, \mathcal{F}_{n}, \mu\right)$ where $\mu$ is the Lebesgue measure is not $\sigma$-finite: any $\mathcal{F}_{n}$ measurable subset of $\Omega \times \mathbb{R}^{d}$ is either of null measure or of infinite measure. Therefore, the concept of $\left(\mathcal{F}_{n}\right)_{n \in \mathbb{N}}$-martingale is ill-defined, see Definition 2.10 and Remark 2.8. Should we attempt to verify whether the martingale defining equality (2.3) hold, we would get either $+\infty$ or 0 on both sides of the equation.

However, the martingale defining equality (2.3) is satisfied if one replaces the Lebesgue integral of $\mathbb{R}^{d}$ by the limit of the mean over a ball as its radius tends to $+\infty$. I.e., we have for all $F$ in $\mathcal{F}_{n}$

$$
\begin{aligned}
\lim _{R \rightarrow+\infty} \frac{1}{|B(\mathbf{0}, R)|} \int_{\Omega} \int_{B(\mathbf{0}, R)} \mathbb{1}\{(\boldsymbol{x}, \boldsymbol{y}) \in F\} u_{0, p_{n}}(\boldsymbol{x}, \boldsymbol{y}) \mathrm{d} \boldsymbol{y} \mathrm{d} \boldsymbol{x}= & \lim _{R \rightarrow+\infty} \frac{1}{|B(\mathbf{0}, R)|} \\
& \times \int_{\Omega} \int_{B(\mathbf{0}, R)} \mathbb{1}\{(\boldsymbol{x}, \boldsymbol{y}) \in F\} u_{0, p_{n+j}}(\boldsymbol{x}, \boldsymbol{y}) \mathrm{d} \boldsymbol{y} \mathrm{d} \boldsymbol{x}
\end{aligned}
$$

where $B(\mathbf{0}, R)$ is the open ball of $\mathbb{R}^{d}$ centered on 0 and of radius $R$ and where $|A|$ is the Lebesgue measure of set $A$. Unfortunately, we were unable to derive a direct convergence result using this pseudo-martingale equality. To proceed further, we need to transform the two-scale limits $u_{0, p_{n}}$ in order to get genuine martingales.

\subsection{Rearrangement of the two-scale limits with integers}

In the previous section, we established a "martingale-like" equality for the two-scale limits $u_{0, p_{n}}$. To get genuine martingales in the sense of Definition 2.10, we need to rearrange the $u_{0, p_{n}}$. While we are unable to prove a convergence for the rearrangement of the two-scale limits presented in this section, the ideas behind this rearrangement provide insight on the next section where we introduce another rearrangement and prove its convergence. 
In this section, we rearrange the $u_{0, p_{n}}$ by introducing a new variable $\boldsymbol{\alpha}$ that belongs to $\mathbb{Z}^{d}$. The rearrangement, denoted by $v_{p_{n}}$, depends on the slow variable $\boldsymbol{x} \in \Omega$, on a fast variable $\boldsymbol{y} \in\left[0, p_{0}{ }^{d}\right.$, and on the new variable $\boldsymbol{\alpha}$. To rearrange the $u_{0, p_{n}}$ into the $v_{p_{n}}$, we subdivide $\Omega \times\left[0, p_{n}\left[{ }^{d}\right.\right.$ into $M_{n}^{d}=\left(p_{n} / p_{0}\right)^{d}$ sets $\Omega \times \prod_{i=1}^{d}\left[\alpha_{i}, \alpha_{i}+p_{0}[\right.$. Each of these sets is the product of $\Omega$ with an hypercube indexed by $\boldsymbol{\alpha}=\left(\alpha_{1}, \ldots, \alpha_{d}\right)$ and we define $v_{p_{n}}(\cdot, \boldsymbol{\alpha}, \cdot)$ as taking in $\Omega \times\left[0, p_{0}\left[{ }^{d}\right.\right.$ the same values $u_{0, p_{n}}$ does in $\Omega \times \prod_{i=1}^{d}\left[\alpha_{i}, \alpha_{i}+p_{0}[\right.$. The variable $\boldsymbol{y}$ represents the position of the fast variable inside each hypercube. I.e., we set:

$$
\begin{aligned}
v_{p_{n}}: \Omega \times \mathbb{Z}^{d} \times Y_{p_{0}} & \rightarrow \mathbb{R}, \\
(\boldsymbol{x}, \boldsymbol{\alpha}, \boldsymbol{y}) & \mapsto u_{0, p_{n}}\left(\boldsymbol{x}, \boldsymbol{y}+p_{0} \boldsymbol{\alpha}\right) .
\end{aligned}
$$

We have the following proposition

Proposition 3.4. For all $n$ in $\mathbb{N}$, for almost all $\boldsymbol{x}$ in $\Omega$ and $\boldsymbol{y}$ in $Y_{p_{0}}$, the $\boldsymbol{\alpha}$-indexed sequence $\left(v_{p_{n}}(\boldsymbol{x}, \boldsymbol{\alpha}, \boldsymbol{y})\right)_{\boldsymbol{\alpha} \in \mathbb{Z}^{d}}$ is $M_{n}$-periodic in each direction of $\boldsymbol{\alpha}$. Moreover:

$$
v_{p_{n}}(\boldsymbol{x}, \boldsymbol{\alpha}, \boldsymbol{y})=\left(\frac{M_{n}}{M_{n+j}}\right)^{d} \sum_{\boldsymbol{\beta} \in \llbracket 0, M_{n+j} / M_{n}-1 \rrbracket^{d}} v_{p_{n+j}}\left(\boldsymbol{x}, \boldsymbol{\alpha}+M_{n} \boldsymbol{\beta}, \boldsymbol{y}\right),
$$

for all $\boldsymbol{\alpha}$ in $\mathbb{Z}^{d}$.

Proof. This is a direct consequence of Proposition 3.3.

This in turn should encourage us to look at the following problem.

Problem 3.5. Let's call "imbricated $\left(M_{n}\right)_{n}$-periodic $d$-dimensional sequences", sequences that satisfy the following properties $\left(t_{n, \boldsymbol{\alpha}}\right)_{n \in \mathbb{N}, \boldsymbol{\alpha} \in \mathbb{Z}^{d}}$ such that

- for all $n$ in $\mathbb{N}$, the $\boldsymbol{\alpha}$-indexed sequence $\left(t_{n, \boldsymbol{\alpha}}\right)_{\boldsymbol{\alpha} \in \mathbb{Z}^{d}}$ is $M_{n}$-periodic in each direction of $\boldsymbol{\alpha}$, i.e. such that for all $n$ in $\mathbb{N}$, for all $\boldsymbol{\alpha}$ in $\mathbb{Z}^{d}$, and for all $\boldsymbol{\beta}$ in $\mathbb{Z}^{d}$ :

$$
t_{n, \boldsymbol{\alpha}}=t_{n, \boldsymbol{\alpha}+M_{n} \boldsymbol{\beta}}
$$

- for all $n$ in $\mathbb{N}$, and for all $\boldsymbol{\alpha}$ in $\mathbb{Z}^{d}$,

$$
t_{n, \boldsymbol{\alpha}}=\left(\frac{M_{n}}{M_{n+j}}\right)^{d} \sum_{\boldsymbol{\beta} \in \llbracket 0, M_{n+j} / M_{n}-1 \rrbracket^{d}} t_{n+j, \boldsymbol{\alpha}+M_{n} \boldsymbol{\beta}}
$$

Study the convergence of $\left(t_{n, \boldsymbol{\alpha}}\right)_{n \in \mathbb{N}, \boldsymbol{\alpha} \in \mathbb{Z}^{d}}$ as $n$ tends to $+\infty$. Under which condition does there exist a sequence $t_{\infty, \alpha}$ such that for all non-negative integers $n$

$$
t_{n, \boldsymbol{\alpha}}=\lim _{N \rightarrow+\infty} \frac{1}{N^{d}} \sum_{\boldsymbol{\beta} \in \llbracket 0, N-1 \rrbracket^{d}} t_{\infty, \boldsymbol{\alpha}+M_{n} \boldsymbol{\beta}}
$$

or such that

$$
t_{n, \boldsymbol{\alpha}}=\lim _{N \rightarrow+\infty} \frac{1}{2^{d} N^{d}} \sum_{\boldsymbol{\beta} \in \llbracket-N, N-1 \rrbracket^{d}} t_{\infty, \boldsymbol{\alpha}+M_{n} \boldsymbol{\beta}}
$$

or both? 
By Proposition 3.4, for almost all $\boldsymbol{x}$ in $\Omega$ and $\boldsymbol{y}$ in $Y_{p_{0}}$, the $\boldsymbol{\alpha}$-indexed sequences $\left(v_{p_{n}}(\boldsymbol{x}, \boldsymbol{\alpha}, \boldsymbol{y})\right)_{\boldsymbol{\alpha} \in \mathbb{Z}^{d}}$ are imbricated $\left(M_{n}\right)_{n}$-periodic $d$-dimensional sequences. Solving Problem 3.5 would be the first step in having a very elegant limit to the $v_{p_{n}}$ as a function defined on $\Omega \times \mathbb{Z}^{d} \times Y_{p_{0}}$. Unfortunately, we do not have an answer for Problem 3.5. While this sequence is morally a martingale with respect to the filtration made of the $\sigma$-fields $\left\{\boldsymbol{\alpha}+M_{n} \mathbb{Z}^{d}, \boldsymbol{\alpha} \in \llbracket 0, M_{n}-1 \rrbracket^{d}\right\}$, it technically is not: we have the same problem we had in the previous section. To conclude with bounded martingales on the convergence, we would need a measure $\mu$ on $\mathbb{Z}^{d}$ such that $\mu\left(\mathbb{Z}^{d}\right)=1$, invariant by translation and such that $\mu\left(m \mathbb{Z}^{d}\right)=1 / m$ whenever $m$ is an integer different from 0 . Such a measure cannot be $\sigma$-additive. If we remove the $\sigma$ additivity constraint, then $\mu$ exists: just set

$$
\mu(A):=\lim _{N \rightarrow+\infty} \frac{\#\left(A \cap \llbracket-N, N \rrbracket^{d}\right)}{(2 N+1)^{d}} .
$$

It is unknown to the author if bounded martingales converge when they are defined on a non $\sigma$-additive measure. To avoid that problem, we introduce, in the next section, a different less natural rearrangement for the $u_{0, p_{n}}$, the shuffle, for which we finally prove a convergence result.

\subsection{Shuffle rearrangement of two-scale limits}

In the previous section, we investigated a rearrangement where the set $\Omega \times\left[0, p_{n}{ }^{d}\right.$ was subdivided into $M_{n}^{d}$ subsets indexed by $\boldsymbol{\alpha} \in \mathbb{Z}^{d}$. In this section, we finally construct a rearrangement, the shuffle, that results in a bounded martingale; thus establishing a convergence result for the $p_{n}$-two-scale limits as $n$ tends to $+\infty$. To do so, we replace the variable $\boldsymbol{\alpha}$ belonging to $\mathbb{Z}^{d}$ with the variable $\boldsymbol{y}^{\prime}$ that belongs to $\left[0,1{ }^{d}\right.$. Like the variable $\boldsymbol{\alpha}$ of the previous section, the variable $\boldsymbol{y}^{\prime}$ indicates which hypercube of edge length $p_{0}$ we consider. The variable $\boldsymbol{y}$ remains unchanged and continue to represent the location inside the hypercube indexed by $\boldsymbol{y}^{\prime}$. We set for $\boldsymbol{x}$ in $\Omega, \boldsymbol{y}$ in $\left[0, p_{0}\right]^{d}$ and $\boldsymbol{y}^{\prime}$ in $[0,1]^{d}$,

$$
w_{n}\left(\boldsymbol{x}, \boldsymbol{y}, \boldsymbol{y}^{\prime}\right):=v_{p_{n}}\left(\boldsymbol{x}, \boldsymbol{\alpha}\left(\boldsymbol{y}^{\prime}\right), \boldsymbol{y}\right),
$$

where $\boldsymbol{\alpha}\left(\boldsymbol{y}^{\prime}\right)_{i}=\left\lfloor M_{n} \boldsymbol{y}_{i}^{\prime}\right\rfloor$ for all integers $i$ in $\llbracket 1, d \rrbracket$. Using Proposition 3.4, we derive that for almost all $\boldsymbol{x}$ in $\Omega$, $\boldsymbol{y}$ in $Y_{p_{0}},(j, n)$ in $\mathbb{N}^{2}$, and $\boldsymbol{\alpha}$ in $\llbracket 0, M_{n}-1 \rrbracket^{d}$

$$
\int_{\prod_{i=1}^{d}\left[\frac{\alpha_{i}}{M_{n}}, \frac{\alpha_{i}+1}{M_{n}}[\right.} w_{n}\left(\boldsymbol{x}, \boldsymbol{y}, \boldsymbol{y}^{\prime}\right) \mathrm{d} \boldsymbol{y}^{\prime}=\sum_{\boldsymbol{\beta} \in \llbracket 0, \frac{M_{n+j}}{M_{n}}-1 \rrbracket^{d}} \int_{\prod_{i=1}^{d}\left[\frac{M_{n} \beta_{i}+\alpha_{i}}{M_{n}+j}, \frac{M_{n} \beta_{i}+\alpha_{i}+1}{M_{n+j}}[\right.} w_{n+j}\left(\boldsymbol{x}, \boldsymbol{y}, \boldsymbol{y}^{\prime}\right) \mathrm{d} \boldsymbol{y}^{\prime} .
$$

To transform the $w_{n}$ into martingales, we need to shuffle the hypercubes as in Figure 1 where, to simplify the drawing, homogenization was only performed on the last component of $\mathbb{R}^{d}$, hence the presence of layers instead of hypercubes. In that figure, we show one step of the rearrangement. As seen in the drawing, each step of the rearrangement is measure preserving, therefore the full rearrangement is also measure preserving. We need $n-1$ such steps to fully rearrange $w_{n}$.

To define rigorously this rearrangement, we begin by defining the function that maps the rearranged layer index onto the unrearranged layer index:

$$
\begin{aligned}
& R_{M, m}: \llbracket 0, M m-1 \rrbracket \rightarrow \llbracket 0, M m-1 \rrbracket \\
& i \mapsto M \cdot(i \quad \bmod m)+\left\lfloor\frac{i}{m}\right\rfloor .
\end{aligned}
$$

The application $R_{M, m}$ maps $k m+j$ to $j M+k$ when $k$ belongs to $\llbracket 0, M-1 \rrbracket$ and $j$ belongs to $\llbracket 0, m-1 \rrbracket$. We also have $R_{M, m} \circ R_{m, M}=R_{m, M} \circ R_{M, m}=$ Id.

Then, we set the function that maps the rearranged layer onto the unrearranged one:

$$
\begin{aligned}
h_{M, m}^{*}: & {[0,1[\rightarrow[0,1[,} \\
y^{\prime} & \mapsto \frac{R_{M, m}\left(\left\lfloor M m y^{\prime}\right\rfloor\right)}{M m}+\left(y^{\prime}-\frac{\left.\left\lfloor M m y^{\prime}\right\rfloor\right)}{M m}\right) .
\end{aligned}
$$




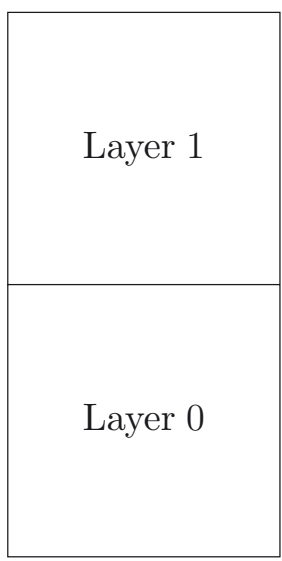

Original $w_{1}$

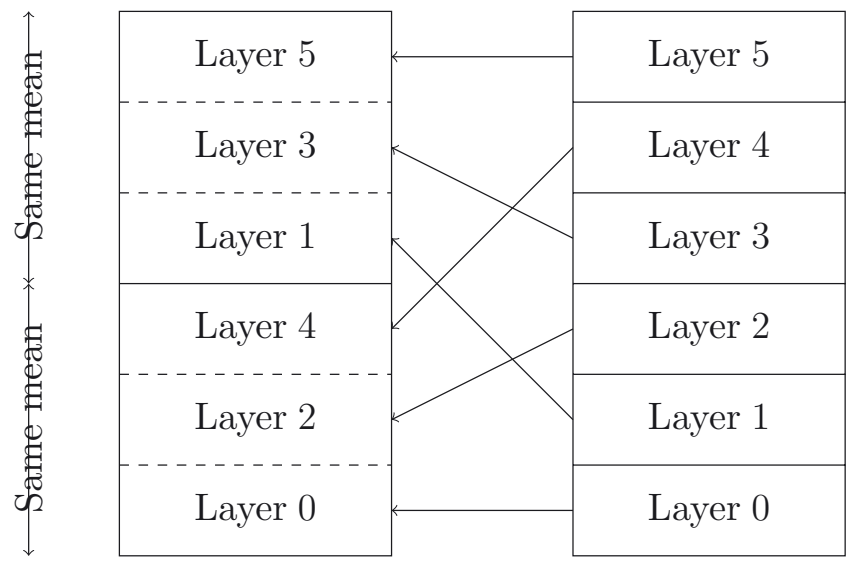

$w_{2}$ after one step

Original $w_{2}$

FiguRE 1. One step of the measure preserving rearrangement $M_{1}=2$ and $M_{2}=6$.

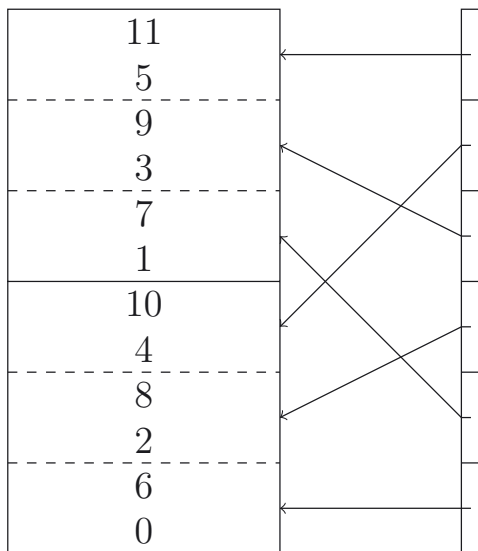

Second step

\begin{tabular}{|l|l|}
\hline & 11 \\
\hline & 10 \\
\hline & 9 \\
\hline
\end{tabular}

First step
Original

Figure 2. Two steps of the measure preserving rearrangement $M_{1}=2, M_{2}=6$ and $M_{3}=12$.

This represents only one step of the rearrangement on one component. For hypercubes, the permutation is the same but is done componentwise: we set

$$
\begin{aligned}
\left.h_{M, m}:\right] 0,1\left[^{d}\right. & \rightarrow] 0,1\left[^{d},\right. \\
\left(y_{1}^{\prime}, \ldots, y_{n}^{\prime}\right) & \mapsto\left(h_{M, m}^{*}\left(y_{1}^{\prime}\right), \ldots, h_{M, m}^{*}\left(y_{n}^{\prime}\right)\right) .
\end{aligned}
$$

And obtain one step of the rearrangement on all $d$ components. For the complete rearrangement on one component, see Figure 2, we set

$$
H_{n}^{*}:=h_{M_{n-1}, m_{n}}^{*} \circ \ldots \circ h_{M_{1}, m_{2}}^{*} \circ h_{M_{0}, m_{1}}^{*} .
$$

To get the complete rearrangement on all components we set

$$
\begin{aligned}
H_{n}:\left[0,1\left[^{d}\right.\right. & \rightarrow\left[0,1\left[^{d},\right.\right. \\
\left(y_{1}^{\prime}, \ldots, y_{n}^{\prime}\right) & \mapsto\left(H_{n}^{*}\left(y_{1}^{\prime}\right), \ldots, H_{n}^{*}\left(y_{n}^{\prime}\right)\right) .
\end{aligned}
$$


We also have

$$
H_{n}=h_{M_{n-1}, m_{n}} \circ \ldots \circ h_{M_{1}, m_{2}} \circ h_{M_{0}, m_{1}} .
$$

The function $H_{n}$ shuffles the hypercubes $\prod_{i=1}^{d}\left[\beta_{i} / M_{n},\left(\beta_{i}+1\right) / M_{n}\left[\right.\right.$, hence we call $H_{n}$ the shuffle function.

Finally, we define

$$
\widetilde{w}_{n}\left(\boldsymbol{x}, \boldsymbol{y}, \boldsymbol{y}^{\prime}\right):=w_{n}\left(\boldsymbol{x}, \boldsymbol{y}, H_{n}\left(\boldsymbol{y}^{\prime}\right)\right) .
$$

This measure preserving rearrangement, the shuffle, is purposefully constructed so the $\widetilde{w}_{n}$ form a martingale for the following filtration of $\sigma$-fields $\mathcal{F}_{n}=\mathcal{B}(\Omega) \times \mathcal{B}\left(\left[0, p_{0}\right]^{d}\right) \times \sigma\left\{\prod_{i=1}^{d}\left[\frac{\beta_{i}}{M_{n}}, \frac{\beta_{i}+1}{M_{n}}\left[, \boldsymbol{\beta} \in \llbracket 0, M_{n}-1 \rrbracket^{d}\right\}\right.\right.$.

Remark 3.6. The above rearrangement of hypercubes is similar to the one used for computing in place the Discrete Fast Fourier Transform: the bit reversal. In the special case where $M_{n}=2^{n}$, the rearrangement simply exchanges layers $i$, i.e., $\left[i / 2^{n},(i+1) / 2^{n}\right.$, and $i^{\prime}$, i.e., $\left[i^{\prime} / 2^{n},\left(i^{\prime}+1\right) / 2^{n}\right.$, when $i$ and $i^{\prime}$ are bit reversal permutations of each other. I.e when $i=\sum_{j=0}^{n-1} b_{j} 2^{j}$ and $i^{\prime}=\sum_{j=0}^{N-1} b_{j} 2^{n-1-j}$.

Remark 3.7. For general $M_{n}$, the rearrangement of hypercubes is also a bit reversal but for a mixed basis. If $\left\lfloor M_{n} y^{\prime}\right\rfloor=\sum_{j=1}^{n} b_{j} M_{j-1}$ with $b_{j}$ in $\llbracket 0, m_{j}-1 \rrbracket$, then

$$
H_{n}^{*}\left(\frac{1}{M_{n}} \sum_{j=1}^{n} b_{j} \frac{M_{n}}{M_{j}}+\left(y^{\prime}-\frac{\left\lfloor M_{n} y^{\prime}\right\rfloor}{M_{n}}\right)\right)=y^{\prime} .
$$

We now state our main result as a self contained theorem.

Theorem 3.8 (Two-Scale Shuffle convergence). Let $\Omega$ be a bounded open domain of $\mathbb{R}^{d}$ with $d \geq 1$. Let $\left(u_{\varepsilon}\right)_{\varepsilon>0}$ be a bounded sequence of functions belonging to $\mathrm{L}^{2}(\Omega)$. Let $\left(p_{n}\right)_{n \in \mathbb{N}}$ be an increasing sequence of positive numbers that satisfy Assumption 3.1. Set for all $n \geq 0 M_{n}:=p_{n} / p_{0}$ and for all $n \geq 1 m_{n}:=p_{n} / p_{n-1}$. Let $\left(\varepsilon_{k}\right)_{k \in \mathbb{N}}$ be a decreasing sequence of positive real numbers converging to 0 such that the sequence $\left(u_{\varepsilon_{k}}\right)_{k \in \mathbb{N}} p_{n}$-two-scale converges to $u_{0, p_{n}}$ for all non-negative integer $n$.

Set

$$
\begin{aligned}
\widetilde{w}_{n}: \Omega \times\left[0, p_{0}\right]^{d} \times[0,1]^{d} & \rightarrow \mathbb{R} \\
\left(\boldsymbol{x}, \boldsymbol{y}, \boldsymbol{y}^{\prime}\right) & \mapsto u_{0, p_{n}}\left(\boldsymbol{x}, p_{0}\left\lfloor M_{n} H_{n}\left(\boldsymbol{y}^{\prime}\right)\right\rfloor+\boldsymbol{y}\right) .
\end{aligned}
$$

where $H_{n}$ is defined by equations (3.4).

Then, the sequence $\widetilde{w}_{n}$ is a bounded martingale in $\mathrm{L}^{2}\left(\Omega \times\left[0, p_{0}\right]^{d} \times[0,1]^{d}\right)$, in the sense of Definition 2.10, for the filtration

$$
\mathcal{F}_{n}=\mathcal{B}(\Omega) \times \mathcal{B}\left(\left[0, p_{0}\right]^{d}\right) \times \sigma\left\{\prod _ { i = 1 } ^ { d } \left[\frac{\beta_{i}}{M_{n}}, \frac{\beta_{i}+1}{M_{n}}\left[, \boldsymbol{\beta} \in \llbracket 0, M_{n}-1 \rrbracket^{d}\right\} .\right.\right.
$$

And, the sequence $\widetilde{w}_{n}$ converges both strongly in $\mathrm{L}^{2}\left(\Omega \times\left[0, p_{0}\right]^{d} \times[0,1]^{d}\right)$ and almost everywhere in $\Omega \times\left[0, p_{0}\right]^{d} \times$ $[0,1]^{d}$ to $\widetilde{w}_{\infty}$, which we call the Two-Scale Shuffle limit. Moreover,

$$
\iiint_{A} \widetilde{w}_{n}\left(\boldsymbol{x}, \boldsymbol{y}, \boldsymbol{y}^{\prime}\right) \mathrm{d} \boldsymbol{y}^{\prime} \mathrm{d} \boldsymbol{y} \mathrm{d} \boldsymbol{x}=\iiint_{A} \widetilde{w}_{\infty}\left(\boldsymbol{x}, \boldsymbol{y}, \boldsymbol{y}^{\prime}\right) \mathrm{d} \boldsymbol{y}^{\prime} \mathrm{d} \boldsymbol{y} \mathrm{d} \boldsymbol{x},
$$

for all sets $A$ in $\mathcal{F}_{n}$. I.e., by Definition 2.7, $\widetilde{w}_{n}=\mathbb{E}\left(\widetilde{w}_{\infty} \mid \mathcal{F}_{n}\right)$.

Proof. The $\widetilde{w}_{n}$ were constructed specifically so as to be a martingale for the filtration (3.6). To prove they are a martingale for the filtration $\left(\mathcal{F}_{n}\right)_{n \in \mathbb{N}}$, we only need to prove that for all non-negative integer $n$, for almost all $\boldsymbol{x}$ in $\Omega$, almost all $\boldsymbol{y}$ in $\left[0, p_{0}\right]^{d}$ and for all $\boldsymbol{\beta}$ in $\llbracket 0, M_{n}-1 \rrbracket^{d}$, we have

$$
\int_{\prod_{i=1}^{d}\left[\frac{\beta_{i}}{M_{n}}, \frac{\beta_{i}+1}{M_{n}}[\right.} \widetilde{w}_{n+1}\left(\boldsymbol{x}, \boldsymbol{y}, \boldsymbol{y}^{\prime}\right) \mathrm{d} \boldsymbol{y}^{\prime}=\int_{\prod_{i=1}^{d}\left[\frac{\beta_{i}}{M_{n}}, \frac{\beta_{i}+1}{M_{n}}[\right.} \widetilde{w}_{n}\left(\boldsymbol{x}, \boldsymbol{y}, \boldsymbol{y}^{\prime}\right) \mathrm{d} \boldsymbol{y}^{\prime} .
$$


I.e., we need to show that

$$
\int_{\prod_{i=1}^{d}\left[\frac{\beta_{i}}{M_{n}}, \frac{\beta_{i}+1}{M_{n}}[\right.} w_{n}\left(\boldsymbol{x}, \boldsymbol{y}, H_{n}\left(\boldsymbol{y}^{\prime}\right)\right) \mathrm{d} \boldsymbol{y}^{\prime}=\int_{\prod_{i=1}^{d}\left[\frac{\beta_{i}}{M_{n}}, \frac{\beta_{i}+1}{M_{n}}[\right.} w_{n+1}\left(\boldsymbol{x}, \boldsymbol{y}, H_{M_{n}, m_{n+1}} \circ H_{n}\left(\boldsymbol{y}^{\prime}\right)\right) \mathrm{d} \boldsymbol{y}^{\prime} .
$$

But $H_{n}$ maps any hypercube $\prod_{i=1}^{d}\left[\beta_{i} / M_{n},\left(\beta_{i}+1\right) / M_{n}\left[\right.\right.$ to another hypercube $\prod_{i=1}^{d}\left[\beta_{i}^{\prime} / M_{n},\left(\beta_{i}^{\prime}+1\right) / M_{n}\left[\right.\right.$ and $H_{n}$ is measure preserving. Therefore, we only need to prove that for almost all $\boldsymbol{x}$ in $\Omega$, almost all $\boldsymbol{y}$ in $\left[0, p_{0}\right]^{d}$ and for all $\boldsymbol{\beta}$ in $\llbracket 0, M_{n}-1 \rrbracket^{d}$

$$
\int_{\prod_{i=1}^{d}\left[\frac{\beta_{i}}{M_{n}}, \frac{\beta_{i}+1}{M_{n}}[\right.} w_{n}\left(\boldsymbol{x}, \boldsymbol{y}, \boldsymbol{y}^{\prime}\right) \mathrm{d} \boldsymbol{y}^{\prime}=\int_{\prod_{i=1}^{d}\left[\frac{\beta_{i}}{M_{n}}, \frac{\beta_{i}+1}{M_{n}}[\right.} w_{n+1}\left(\boldsymbol{x}, \boldsymbol{y}, H_{M_{n}, m_{n+1}}\left(\boldsymbol{y}^{\prime}\right)\right) \mathrm{d} \boldsymbol{y}^{\prime} .
$$

is satisfied. But this equality is equivalent to

$$
\begin{aligned}
\frac{1}{M_{n}^{d}} v_{p_{n}}(\boldsymbol{x}, \boldsymbol{\beta}, \boldsymbol{y}) & =\frac{1}{M_{n+1}^{d}} \sum_{\boldsymbol{\beta}^{\prime} \in \llbracket 0, m_{n+1}-1 \rrbracket^{d}} v_{p_{n+1}}\left(\boldsymbol{x}, R_{M_{n}, m_{n+1}}\left(m_{n+1} \boldsymbol{\beta}+\boldsymbol{\beta}^{\prime}\right), \boldsymbol{y}\right) \\
& =\frac{1}{M_{n+1}^{d}} \sum_{\boldsymbol{\beta}^{\prime} \in \llbracket 0, m_{n+1}-1 \rrbracket^{d}} v_{p_{n+1}}\left(\boldsymbol{x},\left(\boldsymbol{\beta}+\boldsymbol{\beta}^{\prime} M_{n}\right), \boldsymbol{y}\right),
\end{aligned}
$$

which is true by Proposition 3.4. Therefore, the sequence $\widetilde{w}_{n}$ is a martingale for the filtration $\left(\mathcal{F}_{n}\right)_{n \in \mathbb{N}}$.

By Proposition 2.3, this martingale is bounded in $\mathrm{L}^{2}$. It converges both strongly in $\mathrm{L}^{2}\left(\Omega \times\left[0, p_{0}\right]^{d} \times[0,1]^{d}\right)$ and almost everywhere to a function $\widetilde{w}_{\infty}$, see [7], Corollary 7.22.

Corollary 3.9. It is possible to recover $u_{0, p_{n}}$ from the Two-Scale Shuffle limit $\widetilde{w}_{\infty}$. First, for all $\boldsymbol{\beta}$ in $\llbracket 0, M_{n}-$ $1 \rrbracket^{d}$, all $\boldsymbol{y}^{\prime}$ in $\prod_{i=1}^{d}\left[\beta_{i} / M_{n},\left(\beta_{i}+1\right) / M_{n}\left[\right.\right.$, and almost all $(\boldsymbol{x}, \boldsymbol{y})$ in $\Omega \times\left[0, p_{0}\right]^{d}$, we have

$$
\widetilde{w}_{n}\left(\boldsymbol{x}, \boldsymbol{y}, \boldsymbol{y}^{\prime}\right)=M_{n}^{d} \int_{\prod_{i=1}^{d}\left[\frac{\beta_{i}}{M_{n}}, \frac{\beta_{i}+1}{M_{n}}[\right.} \widetilde{w}_{\infty}\left(\boldsymbol{x}, \boldsymbol{y}, \boldsymbol{y}^{\prime}\right) \mathrm{d} \boldsymbol{y}^{\prime}
$$

because $\widetilde{w}_{n}=\mathbb{E}\left(\widetilde{w}_{\infty} \mid \mathcal{F}_{n}\right)$. Since the shuffle function $H_{n}$ is one to one from $\left[0,1{ }^{d}\right.$ to $\left[0,1{ }^{d}\right.$, see Remark 3.7, we have $w_{n}\left(\boldsymbol{x}, \boldsymbol{y}, \boldsymbol{y}^{\prime}\right)=\widetilde{w}_{n}\left(\boldsymbol{x}, \boldsymbol{y}, H_{n}^{-1}\left(\boldsymbol{y}^{\prime}\right)\right)$. Finally, $u_{0, p_{n}}(\boldsymbol{x}, \boldsymbol{y})$ is equal to the constant value taken by $\boldsymbol{y}^{\prime} \mapsto$ $w_{n}\left(\boldsymbol{x}, \boldsymbol{y}-p_{0}\left\lfloor\boldsymbol{y} / p_{0}\right\rfloor, \boldsymbol{y}^{\prime}\right)$ when $\boldsymbol{y}^{\prime}$ belongs to the hypercube $\left[\left\lfloor\boldsymbol{y} / p_{0}\right\rfloor / M_{n},\left(\left\lfloor\boldsymbol{y} / p_{0}\right\rfloor+1\right) / M_{n}[\right.$.

\section{Application: heat equation in multilayers}

In this section, we consider the multilayer heat equation with three spatial dimensions which we homogenize along the vertical space variable, i.e., along the direction perpendicular to the layers.

In [13], the author established the equations satisfied by the two-scale limits of the heat equation in multilayers with transmission conditions between adjacent layers. When the magnitude of the interlayer conductivity between adjacent layers is weak, see [13], Section 6.1, the two-scale limit depends on the number of layers present in the homogenization period, i.e., on the scale factors $p_{n}$. For given values of the slow variables $(\boldsymbol{x}, t)$, the two scale limit is piecewise constant in its scalar fast variable $y$ and takes as many values as there are layers in a single homogenization cell. Our goal is to establish the equation satisfied by the limit of two-scale limits, i.e., the Two-Scale Shuffle limit, as defined in Theorem 3.8.

To do so, we first recall previously known results in Section 4.1, then derive new results using Two-Scale Shuffle Convergence in Section 4.2. 


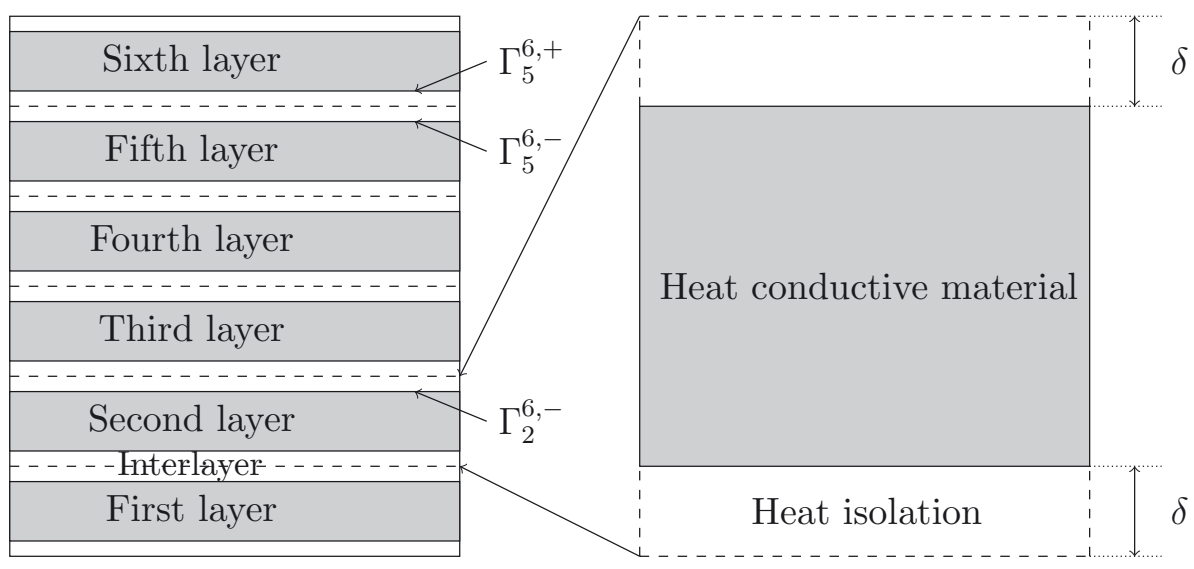

FiguRE 3. The multilayer geometry, six layers: $N=6$.

\subsection{The two-scale limit of the multilayer heat equation}

We start by recalling some results we obtained in [13]. To avoid unnecessary complications, we consider here a simpler problem than the one considered in [13], System (4.1). Let $\Omega$ be $B \times] 0,1[$ where $B$ is a convex bounded open subset of $\mathbb{R}^{2}$ with smooth boundary. Let $\delta, 0<\delta<1 / 2$. Let $I$ be the interval $] \delta, 1-\delta[$. For all $N$, let $I_{N}$ be $\left.\bigcup_{j=0}^{N-1}\right](j+\delta) / N,(j+1-\delta) / N\left[\right.$. Let $\Omega^{N}$ be the domain $B \times I_{N}$. Let $\Gamma_{j}^{N,+}=B \times\{(j+\delta) / N\}$ and $\Gamma_{j}^{N,-}=B \times\{(j-\delta) / N\}$. Let $\Gamma^{N,+}=\bigcup_{j=1}^{N-1} \Gamma_{j}^{N,+}$ and $\Gamma^{N,-}=\bigcup_{j=1}^{N-1} \Gamma_{j}^{N,-}$. Let $\left.\Gamma_{e}=\partial B \times\right] 0,1\left[\cup \Gamma_{0}^{N,+} \cup \Gamma_{N}^{N,-}\right.$. Let $\gamma$ be the application on $\partial \Omega^{N}$ that maps $u$ in $\mathrm{H}^{1}\left(\Omega^{N}\right)$ to its trace on $\partial \Omega^{N}$. Let $\gamma^{\prime}$ be the trace operator swapped between $\Gamma_{j}^{N,+}$ and $\Gamma_{j}^{N,-}:$ i.e., $\gamma^{\prime} u(\hat{\boldsymbol{x}},(j \pm \delta) / N)=\gamma u(\hat{\boldsymbol{x}},(j \mp \delta) / N)$ for all $\hat{\boldsymbol{x}}$ in $B$ and all $j$ in $\llbracket 1, N-1 \rrbracket$. See Figure 3 where we schematized the three dimensional domain $\Omega^{N}$ by projecting it onto the two-dimensional plane.

Let $A, K$ and $J$ be positive reals: $A$ represents the heat conductivity inside $\Omega^{N}$, and $J$ is the magnitude of the surfacic interlayer conductivity. For all positive integer $N$, we consider the multilayer heat equation

$$
\frac{\partial u_{N}}{\partial t}-A \triangle u_{N}=0 \text { in } \Omega^{N} \times \mathbb{R}^{+}
$$

with the boundary conditions

$$
A \frac{\partial u_{N}}{\partial \boldsymbol{\nu}}= \begin{cases}0 & \text { on } \Gamma_{e} \times \mathbb{R}^{+} \\ -\frac{K}{N} \gamma u_{N}+\frac{J}{N}\left(\gamma^{\prime} u_{N}-\gamma u_{N}\right) & \text { on }\left(\Gamma^{N,+} \cup \Gamma^{N,-}\right) \times \mathbb{R}^{+},\end{cases}
$$

and the initial condition

$$
u_{N}(\cdot, 0)=u_{N}^{0} .
$$

We also define the energy

$$
\begin{aligned}
E^{N}(v)= & \frac{A}{2} \int_{\Omega^{N}}\|\nabla v(\boldsymbol{x})\|^{2} \mathrm{~d} \boldsymbol{x}+\frac{K}{2 N} \int_{\Gamma^{N,+} \cup \Gamma^{N,-}}|\gamma v|^{2} \mathrm{~d} \sigma(\boldsymbol{x}) \\
& +\frac{J}{2 N} \sum_{j=1}^{N-1} \int_{B}\left|v\left(\hat{\boldsymbol{x}}, \frac{j+\delta}{N}\right)-v\left(\hat{\boldsymbol{x}}, \frac{j-\delta}{N}\right)\right|^{2} \mathrm{~d} \hat{\boldsymbol{x}},
\end{aligned}
$$

for all $v$ in $H^{1}\left(\Omega^{N}\right)$. We suppose

$$
\sup _{N} E^{N}\left(u_{N}^{0}\right)<+\infty
$$


and denote by $u_{0, M}^{0}$ the $M$-two-scale limit of the initial conditions $u_{N}^{0}$. We have the energy equality

$$
E^{N}\left(u_{N}(\cdot, T)\right)+\int_{0}^{T} \int_{\Omega^{N}}\left|\frac{\partial u_{N}}{\partial t}\right|^{2} \mathrm{~d} \boldsymbol{x} \mathrm{d} t=E^{N}\left(u_{N}(\cdot, T)\right),
$$

for all $T \geq 0$. Because of the energy bound, for all $\boldsymbol{x}$ in $\Omega$ and all $j$ in $\mathbb{Z} / M \mathbb{Z}$, the function $y \mapsto u_{0, M}^{0}(\boldsymbol{x}, y)$ is constant in the interval $] j+\delta, j+1-\delta\left[\right.$. Moreover, that function is $M$-periodic in $y$. We denote by $u_{0, M_{n}, j}^{0}(\boldsymbol{x})$ the value taken by the function $y \mapsto u_{0, M}^{0}(\boldsymbol{x}, y)$ in the interval $] j+\delta, j+1-\delta[$.

Using two-scale convergence $[1,10]$ and its variant on periodic surfaces $[2,8,9]$, the properties of the two-scale limit of $\left(u^{N}\right)_{N \in \mathbb{N}}$, solutions to the multilayer heat system (4.1) with $J \neq 0$, were established in [13], Theorem 6.1.

Let $\left(M_{n}\right)_{n \geq 0}$ be a sequence of positive integers such that $M_{0}=1$ and such that $M_{n+1}$ is always an entire multiple of $M_{n}$. For all $(\boldsymbol{x}, t)$ in $\Omega \times \mathbb{R}^{+}$, the $M_{n}$-two-scale limit $y \mapsto u_{0, M_{n}}(\boldsymbol{x}, t, y)$ takes $M_{n}$ values: it is constant in each interval $] j+\delta, j+1-\delta\left[\right.$. For $j$ in $\mathbb{Z} / M_{n} \mathbb{Z}$, we note $u_{0, M_{n}, j}(\boldsymbol{x}, t)$ the value of $u_{0, M_{n}}(\boldsymbol{x}, t, \cdot)$ in this interval. We have $u_{0, M_{n}, j+M_{n}}(\boldsymbol{x}, t)=u_{0, M_{n}, j}(\boldsymbol{x}, t)$. These functions satisfy, for all $j$ in $\mathbb{Z} / M_{n} \mathbb{Z}$, the weak formulation of

$$
\frac{\partial u_{0, M_{n}, j}}{\partial t}-A \triangle_{\mathrm{T}} u_{0, M_{n}, j}+\frac{2 K}{1-2 \delta} u_{0, M_{n}, j}+\frac{J}{1-2 \delta}\left(2 u_{0, M_{n}, j}-u_{0, M_{n}, j+1}-u_{0, M_{n}, j-1}\right)=0
$$

in $\Omega \times \mathbb{R}^{+}$, and where $\triangle_{\mathrm{T}}=\frac{\partial^{2}}{\partial x_{1}^{2}}+\frac{\partial^{2}}{\partial x_{2}^{2}}, \nabla_{\mathrm{T}}=\left[\frac{\partial}{\partial x_{1}}, \frac{\partial}{\partial x_{2}}\right]^{\mathrm{T}}$. And with boundary conditions

$$
\frac{\partial u_{0, M_{n}, j}}{\partial \boldsymbol{\nu}}=0 \text { on }(\partial B \times] 0,1[) \times \mathbb{R}^{+},
$$

and initial condition

$$
\left.u_{0, M_{n}, j}(\cdot, 0)=u_{0, M_{n}, j}^{0} \text { in } B \times\right] 0,1[.
$$

We have recalled previously known results on the properties of the two-scale limits of the multilayer heat equation. In the next section, we establish the properties satisfied by the Two-Scale Shuffle limit of the multilayer heat equation.

\subsection{The Two-Scale Shuffle limit of the multilayer heat equation}

We now use Theorem 3.8 to have the two-scale limits themselves converge. We choose $M_{n}=2^{n}$ to avoid complications at first. We establish the following:

Theorem 4.1. Let $\widetilde{w}_{\infty}$ be the Two-Scale Shuffle limit defined from $\left(u_{0,2^{n}}\right)_{n \in \mathbb{N}}$ as in Theorem 3.8. For all $(\boldsymbol{x}, t)$ in $\Omega \times \mathbb{R}^{+}$, and $y^{\prime}$ in $[0,1]$. The function $\widetilde{w}_{\infty}\left(\boldsymbol{x}, t, \cdot, y^{\prime}\right)$ is constant in the interval $] \delta, 1-\delta[$. If we denote by $\widetilde{w}_{\infty}\left(\boldsymbol{x}, t, y^{\prime}\right)$ the value of $\widetilde{w}_{\infty}\left(\boldsymbol{x}, t, \cdot, y^{\prime}\right)$ inside the interval $] \delta, 1-\delta\left[\right.$, the Two-Scale Shuffle limit $\widetilde{w}_{\infty}$ is a weak solution to:

$$
\begin{aligned}
\frac{\partial \widetilde{w}_{\infty}}{\partial t}\left(\boldsymbol{x}, t, y^{\prime}\right)-A \triangle_{\mathrm{T}} \widetilde{w}_{\infty}\left(\boldsymbol{x}, t, y^{\prime}\right)+\frac{2 K}{1-2 \delta} & \widetilde{w}_{\infty}\left(\boldsymbol{x}, t, y^{\prime}\right)+\frac{J}{1-2 \delta} \\
& \times\left(2 \widetilde{w}_{\infty}\left(\boldsymbol{x}, t, y^{\prime}\right)-\widetilde{w}_{\infty}\left(\boldsymbol{x}, t, \tau^{+}\left(y^{\prime}\right)\right)-\widetilde{w}_{\infty}\left(\boldsymbol{x}, t, \tau^{-}\left(y^{\prime}\right)\right)=0,\right.
\end{aligned}
$$

in $\left.\Omega \times \mathbb{R}^{+} \times\right] 0,1[$, and where, for all non-negative integers $j$ :

$$
\begin{aligned}
& \tau^{+}\left(y^{\prime}\right)=y^{\prime}+3 \cdot 2^{-(j+1)}-1 \text { when } 1-2^{-j} \leq y^{\prime}<1-2^{-(j+1)}, \\
& \tau^{-}\left(y^{\prime}\right)=y^{\prime}-3 \cdot 2^{-(j+1)}+1 \text { when } 2^{-(j+1)} \leq y^{\prime}<2^{-j}
\end{aligned}
$$

with boundary conditions

$$
\left.\frac{\partial \widetilde{w}_{\infty}}{\partial \boldsymbol{\nu}}=0 \text { on }(\partial B \times] 0,1[) \times \mathbb{R}^{+} \times\right] 0,1[,
$$


and initial condition

$$
\widetilde{w}_{\infty}(\cdot, 0, \cdot)=\widetilde{w}_{\infty}^{0}
$$

where $\widetilde{w}_{\infty}^{0}$ is the Two-Scale Shuffle Limit of the initial conditions and where, as an abuse of notations, we denote by $\widetilde{w}_{\infty}^{0}\left(\boldsymbol{x}, t, y^{\prime}\right)$ the constant value taken by $y \mapsto \widetilde{w}_{\infty}^{0}\left(\boldsymbol{x}, t, y, y^{\prime}\right)$ in the interval $] \delta, 1-\delta[$.

Proof. Let $n$ in $\mathbb{N}$ and $\beta$ belongs to $\llbracket 0,2^{n}-1 \rrbracket$. Let the $w_{n}$ be defined as in Section 3.3 , and the $\widetilde{w}_{n}$ be defined from the $2^{n}$-two scale limits $u_{0,2^{n}}$ as in Theorem 3.8. Both the $\widetilde{w}_{n}$ and the $w_{n}$ are functions defined over $\left.(B \times] 0,1[) \times \mathbb{R}^{+} \times\right] 0,1[\times] 0,1\left[\right.$. For all $(\boldsymbol{x}, t)$ in $(B \times] 0,1[) \times \mathbb{R}^{+}$, and $y^{\prime}$ in $] 0,1\left[\right.$, the application $y \mapsto \widetilde{w}_{n}\left(\boldsymbol{x}, t, y, y^{\prime}\right)$ is constant on $] \delta, 1-\delta\left[\right.$. As an abuse of notations, let's also denote by $\widetilde{w}_{n}\left(\boldsymbol{x}, t, y^{\prime}\right)$ the value of $\widetilde{w}_{n}\left(\boldsymbol{x}, t, y^{\prime}, y\right)$ when $y$ belongs to $] \delta, 1-\delta\left[\right.$. We use the same abuse of notations for the $w_{n}$. Consider a test function $\varphi$ belonging to $\mathcal{C}^{\infty}\left(\bar{\Omega} \times \mathbb{R}^{+}\right)$. Set $\psi(\boldsymbol{x}, t, y)=\varphi(x, t) \mathbb{1}\left\{y \in\left[\frac{\beta}{2^{n}}, \frac{\beta+1}{2^{n}}[\}\right.\right.$. Let $Q_{T}=\Omega \times \mathbb{R}^{+}$. Then, since the $u_{0,2^{n}, j}$ satisfy the weak formulation of (4.2), we have:

$$
\begin{array}{r}
\iint_{Q_{T}} \int_{\left[\frac{\beta}{2^{n}}, \frac{\beta+1}{2^{n}}[\right.} \frac{\partial w_{n}}{\partial t}\left(\boldsymbol{x}, t, y^{\prime}\right) \cdot \varphi(\boldsymbol{x}, t) \mathrm{d} y^{\prime} \mathrm{d} \boldsymbol{x} \mathrm{d} t+A \iint_{Q_{T}} \int_{\left[\frac{\beta}{2^{n}}, \frac{\beta+1}{2^{n}}[\right.} \nabla_{\mathrm{T}} w_{n}\left(\boldsymbol{x}, t, y^{\prime}\right) \cdot \nabla_{\mathrm{T}} \varphi(\boldsymbol{x}, t) \mathrm{d} y^{\prime} \mathrm{d} \boldsymbol{x} \mathrm{d} t \\
+\frac{2 K}{1-2 \delta} \iint_{Q_{T}} \int_{\left[\frac{\beta}{2^{n}}, \frac{\beta+1}{2^{n}}[\right.} w_{n}\left(\boldsymbol{x}, t, y^{\prime}\right) \cdot \varphi(\boldsymbol{x}, t) \mathrm{d} y^{\prime} \mathrm{d} \boldsymbol{x} \mathrm{d} t+\frac{J}{1-2 \delta} \iint_{q_{T}} \int_{\left[\frac{\beta}{2^{n}}, \frac{\beta+1}{2^{n}}[\right.} 2 w_{n}\left(\boldsymbol{x}, t, y^{\prime}\right) \cdot \varphi(\boldsymbol{x}, t) \mathrm{d} y^{\prime} \mathrm{d} \boldsymbol{x} \mathrm{d} t \\
\quad-\frac{J}{1-2 \delta} \iint_{q_{T}} \int_{\left[\frac{\beta}{2^{n}}, \frac{\beta+1}{2^{n}}[\right.}\left(w_{n}\left(\boldsymbol{x}, t, y^{\prime}+2^{-n}\right)+w_{n}\left(\boldsymbol{x}, t, y^{\prime}-2^{-n}\right)\right) \cdot \varphi(\boldsymbol{x}, t) \mathrm{d} y^{\prime} \mathrm{d} \boldsymbol{x} \mathrm{d} t=0
\end{array}
$$

where, to simplify notations, we consider the function $w_{n}$ to be 1 -periodic in $y^{\prime}$. Therefore, for all $\beta$ in $\llbracket 0,2^{n}-1 \rrbracket$,

$$
\begin{gathered}
\iint_{Q_{T}} \int_{\left[\frac{\beta}{2^{n}}, \frac{\beta+1}{2^{n}}[\right.} \frac{\partial \widetilde{w}_{n}}{\partial t}\left(\boldsymbol{x}, t, y^{\prime}\right) \cdot \varphi(\boldsymbol{x}, t) \mathrm{d} y^{\prime} \mathrm{d} \boldsymbol{x} \mathrm{d} t+A \iint_{Q_{T}} \int_{\left[\frac{\beta}{2^{n}}, \frac{\beta+1}{2^{n}}[\right.} \nabla_{\mathrm{T}} \widetilde{w}_{n}\left(\boldsymbol{x}, t, y^{\prime}\right) \cdot \nabla_{\mathrm{T}} \varphi(\boldsymbol{x}, t) \mathrm{d} y^{\prime} \mathrm{d} \boldsymbol{x} \mathrm{d} t \\
+\frac{2 K}{1-2 \delta} \iint_{Q_{T}} \int_{\left[\frac{\beta}{2^{n}}, \frac{\beta+1}{2^{n}}[\right.} \widetilde{w}_{n}\left(\boldsymbol{x}, t, y^{\prime}\right) \cdot \varphi(\boldsymbol{x}, t) \mathrm{d} y^{\prime} \mathrm{d} \boldsymbol{x} \mathrm{d} t+\frac{J}{1-2 \delta} \iint_{Q_{T}} \int_{\left[\frac{\beta}{2^{n}}, \frac{\beta+1}{2^{n}}[\right.} 2 \widetilde{w}_{n}\left(\boldsymbol{x}, t, y^{\prime}\right) \cdot \varphi(\boldsymbol{x}, t) \mathrm{d} y^{\prime} \mathrm{d} \boldsymbol{x} \mathrm{d} t \\
-\frac{J}{1-2 \delta} \iint_{Q_{T}} \int_{\left[\frac{\beta}{2^{n}}, \frac{\beta+1}{2^{n}}[\right.} \widetilde{w}_{n}\left(\boldsymbol{x}, t, H_{n}^{*-1}\left(H_{n}^{*}\left(y^{\prime}\right)+2^{-n}\right)\right) \cdot \varphi(\boldsymbol{x}, t) \mathrm{d} y^{\prime} \mathrm{d} \boldsymbol{x} \mathrm{d} t \\
\quad-\frac{J}{1-2 \delta} \iint_{Q_{T}} \int_{\left[\frac{\beta}{2^{n}}, \frac{\beta+1}{2^{n}}[\right.} \widetilde{w}_{n}\left(\boldsymbol{x}, t, H_{n}^{*-1}\left(H_{n}^{*}\left(y^{\prime}\right)-2^{-n}\right)\right) \cdot \varphi(\boldsymbol{x}, t) \mathrm{d} y^{\prime} \mathrm{d} \boldsymbol{x} \mathrm{d} t=0 .
\end{gathered}
$$

Here $H_{n}^{*}$ is simply the bit reversal of the first $n$ coefficients in the binary expansion. Thus:

$$
H_{n}^{*-1}\left(H_{n}^{*}\left(y^{\prime}+2^{-n}\right)\right)= \begin{cases}y^{\prime}+3 \cdot 2^{-(j+1)}-1 & \text { if } 1-2^{-j} \leq y^{\prime}<1-2^{-(j+1)} \\ & \text { for } 0 \leq j \leq n-1 \\ y^{\prime}-1+2^{-n} & \text { if } 1-2^{-n} \leq y^{\prime}<1\end{cases}
$$

And

$$
H_{n}^{*-1}\left(H_{n}^{*}\left(y^{\prime}\right)-2^{-n}\right)= \begin{cases}y^{\prime}-3 \cdot 2^{-(j+1)}+1 & \text { if } 2^{-(j+1)} \leq y^{\prime}<2^{-j} \\ & \text { for } 0 \leq j \leq n-1 \\ y^{\prime}+1-2^{-n} & \text { if } 0 \leq y^{\prime}<2^{-n}\end{cases}
$$


Since $\varphi(\boldsymbol{x}, t) \mathbb{1}\left\{y^{\prime} \in\left[\frac{\beta}{2^{n}}, \frac{\beta+1}{2^{n}}[\}\right.\right.$ is $\mathcal{F}_{n}$-measurable and $\widetilde{w}_{n}=\mathbb{E}\left(\widetilde{w}_{\infty} \mid \mathcal{F}_{n}\right)$, equality (4.4) remains valid after replacing $\widetilde{w}_{n}$ by $\widetilde{w}_{\infty}$. Therefore,

$$
\begin{aligned}
& \iint_{Q_{T}} \int_{\left[\frac{\beta}{2^{n}}, \frac{\beta+1}{2^{n}}[\right.} \frac{\partial \widetilde{w}_{\infty}}{\partial t}\left(\boldsymbol{x}, t, y^{\prime}\right) \cdot \varphi(\boldsymbol{x}, t) \mathrm{d} y^{\prime} \mathrm{d} \boldsymbol{x} \mathrm{d} t+A \iint_{Q_{T}} \int_{\left[\frac{\beta}{2^{n}}, \frac{\beta+1}{2^{n}}[\right.} \nabla_{\mathrm{T}} \widetilde{w}_{\infty}\left(\boldsymbol{x}, t, y^{\prime}\right) \cdot \nabla_{\mathrm{T}} \varphi(\boldsymbol{x}, t) \mathrm{d} y^{\prime} \mathrm{d} \boldsymbol{x} \mathrm{d} t \\
& +\frac{2 K}{1-2 \delta} \iint_{Q_{T}} \int_{\left[\frac{\beta}{2^{n}}, \frac{\beta+1}{2^{n}}[\right.} \widetilde{w}_{\infty}\left(\boldsymbol{x}, t, y^{\prime}\right) \cdot \varphi(\boldsymbol{x}, t) \mathrm{d} y^{\prime} \mathrm{d} \boldsymbol{x} \mathrm{d} t+\frac{J}{1-2 \delta} \iint_{q_{T}} \int_{\left[\frac{\beta}{2^{n}}, \frac{\beta+1}{2^{n}}[\right.} 2 \widetilde{w}_{\infty}\left(\boldsymbol{x}, t, y^{\prime}\right) \cdot \varphi(\boldsymbol{x}, t) \mathrm{d} y^{\prime} \mathrm{d} \boldsymbol{x} \mathrm{d} t \\
& -\frac{J}{1-2 \delta} \iint_{q_{T}} \int_{\left[\frac{\beta}{2^{n}}, \frac{\beta+1}{2^{n}}[\right.}\left(\widetilde{w}_{\infty}\left(\boldsymbol{x}, t, \tau^{+}\left(y^{\prime}\right)\right)+\widetilde{w}_{\infty}\left(\boldsymbol{x}, t, \tau^{-}\left(y^{\prime}\right)\right)\right) \cdot \varphi(\boldsymbol{x}, t) \mathrm{d} y^{\prime} \mathrm{d} \boldsymbol{x} \mathrm{d} t=0
\end{aligned}
$$

for all $n$ in $\mathbb{N}$ and $\beta$ in $\llbracket 1,2^{n}-2 \rrbracket$. Choose $y^{\prime}$ in $] 0,1\left[\right.$, for any positive integer $n$, set $\beta=\left\lfloor 2^{n} y^{\prime}\right\rfloor$ and take the limit in the above equality divided by $2^{-n}$ as $n$ tends to $+\infty$.

If instead of setting $M_{n}=2^{n}$, we consider a general sequence $\left(M_{n}\right)_{n \in \mathbb{N}}$, the same reasonning holds. When $M_{n}$ is $2^{n}$, the shuffling of layers is the bit reversal of the first $n$ coefficients of the binary representation of $y$, thus involutive. This is not the case for general $M_{n}$ and we must use Remark 3.7. Therefore, utmost care must be taken to compute the analogues of (4.5) and (4.6). We provide the limit in the general case without proof. In that case, we have

$$
\begin{aligned}
& H_{n}^{*-1}\left(H_{n}^{*}\left(y^{\prime}\right)+\frac{1}{M_{n}}\right)= \begin{cases}y^{\prime}-\sum_{l=1}^{j} \frac{1}{M_{l}}+\frac{1}{M_{j+1}} & \text { if } 1-\frac{1}{M_{j}} \leq y^{\prime}<1-\frac{1}{M_{j+1}} \\
y^{\prime}-1+\frac{1}{M_{n}} & \text { for } 0 \leq j \leq n-1,\end{cases} \\
& H_{n}^{*-1}\left(H_{n}^{*}\left(y^{\prime}\right)-\frac{1}{M_{n}}\right)= \begin{cases}y^{\prime}+\sum_{l=1}^{j} \frac{1}{M_{l}}-\frac{1}{M_{j+1}} & \text { if } \frac{1}{M_{j}} \leq y^{\prime}<\frac{1}{M_{j+1}} \\
y^{\prime}+1-\frac{1}{M_{n}} & \text { for } 0 \leq j \leq n-1\end{cases}
\end{aligned}
$$

and the limit equation (4.3a) remains valid if we set instead

$$
\begin{aligned}
& \tau^{+}\left(y^{\prime}\right)=y^{\prime}-\sum_{l=1}^{j} \frac{1}{M_{l}}+\frac{1}{M_{j+1}} \text { when } 1-\frac{1}{M_{j}} \leq y^{\prime}<1-\frac{1}{M_{j+1}} \\
& \tau^{-}\left(y^{\prime}\right)=y^{\prime}+\sum_{l=1}^{j} \frac{1}{M_{l}}-\frac{1}{M_{j+1}} \text { when } \frac{1}{M_{j+1}} \leq y^{\prime}<\frac{1}{M_{j}},
\end{aligned}
$$

for all non-negative integer $j$.

\section{Conclusion}

We have proven in this paper, see Theorem 3.8, that the two-scale limits of a given sequence of functions, computed for periods that are entire multiple of the previous ones, form a bounded martingale and thus converge both strongly in $\mathrm{L}^{2}$ and almost everywhere. From the limit, called the Two-Scale Shuffle limit, one can recover any element in the sequence of two-scale limits: this limit contains all the information contained in the whole sequence of two-scale limits, see Corollary 3.9. For a good choice of increasing periods, this limits captures everything that happens at any length scale that is an entire multiple of $\varepsilon$.

Unfortunately, this limit does not capture all phenomena with a period linear in $\varepsilon$ : it cannot capture phenomena with an irrational scale factor. The construction of the martingale depends on the assumption that $p_{n+1}$ is always an entire multiple of $p_{n}$. If there are two interesting scales whose ratio is irrational then no choice of periodic scale carry the information for both scales. 
We applied the notion of the Two-Scale Shuffle limit to the heat equation on multilayers with transmission conditions between adjacent layers. We then considered the solutions to these equations and established the equation satisfied by their Two-Scale Shuffle limit.

To establish the convergence of the two-scale limit, we used the shuffle of hypercubes described in Section 3.3. Unfortunately, because of this shuffle, it is not easy to reach an intuitive understanding of the Two-Scale Shuffle limit. Results on the existence of the limit in the setting of Section 3.2 would not have that drawback. Solving Problem 3.5 would be a first step to obtain a limit in this setting.

\section{REFERENCES}

[1] G. Allaire, Homogenization and two-scale convergence. SIAM J. Math. Anal. 23 (1992) 1482-1518.

[2] G. Allaire, Alain Damlamian and Ulrich Hornung, Two-scale convergence on periodic surfaces and applications, in Proc. International Conference on Mathematical Modelling of Flow through Porous Media, edited by A. Bourgeat et al. World Scientific Pub., Singapore (1995) 15-25.

[3] G. Allaire and C. Conca, Bloch wave homogenization and spectral asymptotic analysis. J. Math. Pures Appl. 77 (1998) $153-208$.

[4] G. Ben Arous and H. Owhadi, Multiscale homogenization with bounded ratios and anomalous slow diffusion. Commun. Pure Appl. Math. 56 (2003) 80-113.

[5] D. Cioranescu and J. Saint Jean Paulin, Homogenization in open sets with holes. J. Math. Anal. Appl. 71 (1979) $590-607$.

[6] A. Damlamian and P. Donato, Which sequences of holes are admissible for periodic homogenization with Neumann boundary condition? ESAIM: COCV 8 (2002) 555-585.

[7] O. Kallenberg, Foundations of Modern Probability. Probability and its applications, 2nd edition. Springer (2002).

[8] M. Neuss-Radu, Homogenization techniques. Diplomaarbeit. University of Heidelberg (1992).

[9] M. Neuss-Radu, Some extensions of two-scale convergence. C. R. Acad. Sci. Paris Sér. I Math. 322 (1996) 899-904.

[10] G. Nguetseng, A general convergence result for a functional related to the theory of homogenization. SIAM J. Math. Anal. 20 (1989) 608-623.

[11] W. Rudin, Real and Complex Analysis. 3rd edition. McGraw-Hill, Inc. (1987).

[12] K. Santugini-Repiquet, Homogenization of ferromagnetic multilayers in the presence of surface energies. ESAIM: COCV 13 (2007) 305-330.

[13] K. Santugini-Repiquet, Homogenization of the heat equation in multilayers with interlayer conduction. Proc. Roy. Soc. Edinburgh Sect. A 137 (2007) 147-181. 